\title{
Vibro-Electrical Behavior of a Viscoelastic Piezo-Nanowire in an Elastic Substrate Considering Stress Nonlocality and Microstructural Size-Dependent Effects
}

\author{
Mohammad Malikan \\ Department of Mechanical Engineering, Faculty of Engineering, Islamic Azad University, Mashhad Branch, \\ Mashhad, Iran \\ E-mail address: $\underline{\text { mohammad.malikan@yahoo.com }}$ \\ ORCID numbers of authors: \\ 0000-0001-7356-2168
}

Received date: 18.05 .2019

Accepted date: 25.06 .2019

\begin{abstract}
This research deals with dynamic response of a Polymer/BaTiO ${ }_{3}$ nanowire including viscosity influences. The wire is also impressed by a longitudinal electric field. Hamilton's principle, Lagrangian strains and a refined higher-order beam theory are combined together in order to derive equations of motion. By combining nonlocality and small size effects of a unique model into the derived equations, the couple relations which describe nanosize behavior in a small scale are presented. By employing an analytical approach, the fundamental natural frequencies are calculated numerically. The important results display that the effect of internal viscosity and nonlocality whenever the nanowire is very large are pointless.
\end{abstract}

Keywords: Dynamics response; Piezo-nanowires; Viscosity; Nonlocal theory of strain gradient; Analytical approach

\section{Introduction and Literature review}

In continuation of discovering of carbon nanotubes by Ijimia in 1991, a wide attention has been paid to the other one-dimensional nanomaterials for example; nanobelts, nanorods and nanowires [1]. Quasi aforementioned one-dimensional materials are a new group which in recent years have been presented in many scientific research. It was proved that these materials with a non-carbonic base show the amazing optic, thermal and mechanical characteristics and are utilized as main structural group in nanoscience and nanotechnology for equipment such as biological and chemical sensors, field effect transistors and logic circuits [2].

Paying attention to the new shape of nanostructures, namely nanowires, has been doubled after year 2000. The structures with thickness or diameter of a few tens of nanometers and even smaller and also non-limited length can be defined as nanowires. The nanowires' cross section might be cylindrical, hexagonal, polygons or etc. with regard to their crystallography [3]. The nanowires' length can be variable from a few tens of nanometers to micron or even millimeter. These nanostructures regarding their special properties can be used in the new 
electronic parts. Because developments and progressives in the electronic industries are due to the decreasing of their parts [4].

One of the most attractive nanostructures can be nanowires made of metals due to their unique properties, which lead to their various applications. Nanowires can be used on computers and other calculating devices. Nanoscale wires are required to achieve complex nanoscale electronics. In addition, the nanowires itself can be the basis of electronic components, such as memory [4]. Proper understanding of the properties, applications, and methods of making nanowires is very important because it will enable researchers to construct nanowires with controlled properties and dimensions and can easily adapt them to fit the structural elements in minimizing electrical and electronic equipment [3].

Theoretical investigations on the prediction of mechanical response of nanowires are rare and there have been a few studies about nanowires. Kiani [5] analyzed dynamically a nanowire exposed to a longitudinal magnetic shock. He assumed wires on an elastic foundation and used nonlocal continuum theory to study quantum effects. He also solved the obtained equations of motion by a semi-analytical approach. In another study, Kiani [6] examined a double current-carrying nanowire exposed to a longitudinal magnetic field based on a new integro-surface energy method. Pishkenari et al. [7] studied transverse natural frequencies of a silicon nanowire using atomistic simulation method. To model the nanoscale, they proposed a new continuum model at which surface stress and surface elasticity were considered by both Timoshenko and Euler-Bernoulli beam models. Their outcomes estimated the results for Timoshenko approach including surface effects are matched with MD results. Fu and Zhang [8] reported stability critical conditions and free torsional natural frequencies of an established continuum core-shell nanowire which included weak interfaces based on the surface elasticity. Dynamic buckling and free vibrations of a nanowire with an initial deformation considering surface effects were investigated by Kiani [9]. The nanowire was placed in an axial magnetic surround and frequencies of vibration were computed analytically. Gongbai et al. [10] investigated harmonic and transient response of an atomic nanowire made of silicon. Zhoua et al. [11] formulated nonlinear resonance of a $\mathrm{ZnO}$ piezo-nanowire derived by an electric field. Their results agreed with the experimental outcomes. Zhang et al. [12] combined the Euler-Bernoulli approach and a high-order surface stress in order to study transverse vibrations of a nanowire placed in a polymer foundation exposed to an axial compressive force. The pivot boundary condition was satisfied analytically based on a closedform solution. Li et al. [13] demonstrated three different elastic substrates for analysis free natural and excitation frequencies of a nanowire. The governing equations were derived regarding a surface elasticity and solved respecting to various boundary conditions. Su et al. [14] addressed small scale effects for considering mechanically transverse response of several nanostructures with one-dimension like nanoropes and nanowires. They presented the strain gradient model to examine small size influences and obtained constitutive equations on the basis of classical beam theory. Finally they computed free vibrations and buckling of the models under several edge conditions and validated the numerical results with the experimental tests. Samaei et al. [15] presented free vibration of a piezo-nanowire under an electric field for which surface effects addressed size influences. The simply-supported rectangular wire was modeled analytically on the basis of both Timoshenko and EulerBernoulli beam approaches. Their outcomes showed that the shear deformations imposed a remarkable impact on the dynamics characteristics of the nanowire. Gheshlaghi and Hasheminejad [16] analyzed dynamically a piezo-nanowire included both nonlocal and surface elasticity effects based on the classic beam theory. An explicit solution technique was devoted to compute natural frequencies of the wire. Kiani also published some other research works related to the nanowires [17-19]. Mercan et al. [20] modeled stability of a Silicon 
Carbide nanowire on the basis of a higher-order elasticity theory. Mercan and Civalek [21] analyzed dynamically a micro/nanowire based on the finite element model. On the other hand, Numanoglu et al. [22] examined natural frequencies of an Au nanowire based on the continuum elasticity approach. In terms of nanostructures analyses, there are a wide range of valuable published research in several conditions [23-65].

Heretofore by review the literature, it is clear that the nanowires have been examined rarely which the most important ones are above mentioned. However within the published research there is no one in which a nanowire has viscoelastic influences. There is no doubt that considering a viscoelastic piezo-nanowire can lead to attractive results. Additionally, the Polymer $/ \mathrm{BaTiO}_{3}$ nanowire has been rarely investigated. Therefore, in this paper it is tried to show a new schema for analysis of nanowires. To this, a modified beam model is employed from which one equation is obtained only whenever the classical mechanics is taken into consideration. To be the small scale influences taken into account, nonlocal strain gradient theory is applied. This model examines both size-dependent and nonlocality characteristics. In order to simply transfer the partial differential equation into the algebraic one the Navier method is utilized. This method fully satisfies pivot boundary condition. Afterwards, the outcomes for variety of cases would be depicted numerically.

\section{Theoretical Modelling}

$\mathrm{A} \mathrm{BaTiO}_{3}$ nanowire is lied in a polymer matrix and displayed by Figure below. Cartesian coordinate is connected to the center of the wire (x) and along the upper quadrant of its diameter (z).

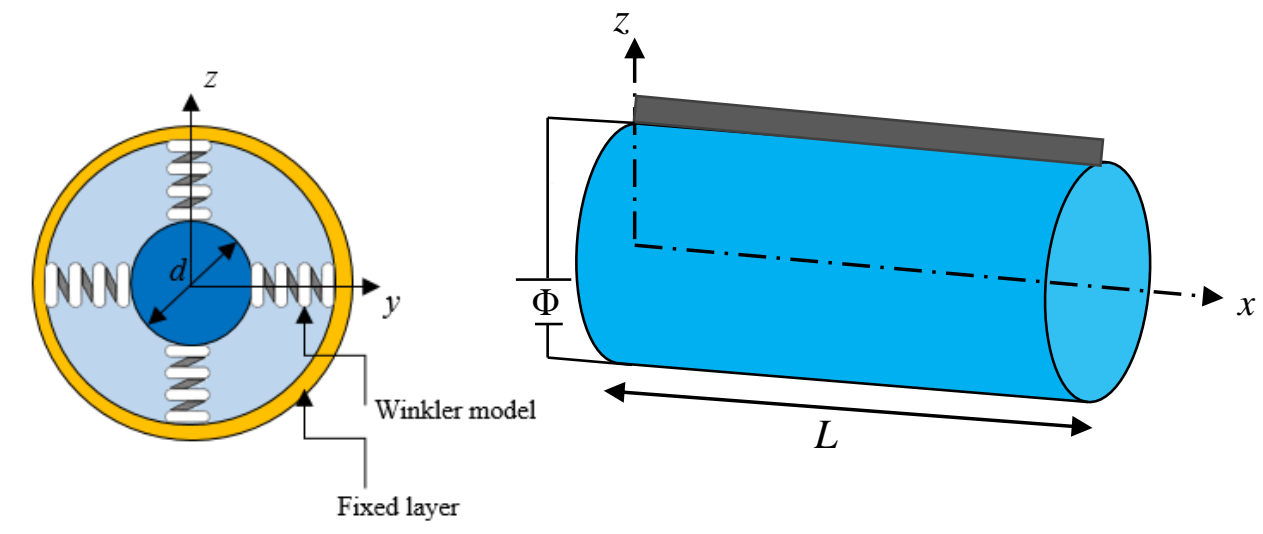

Fig. 1. A Polymer/BaTiO 3 nanowire in a Cartesian coordinate system

Regarding the refined shear deformation beam theory [48], the given displacement field is described as

$$
\left\{\begin{array}{l}
U(x, z, t) \\
W(x, z, t)
\end{array}\right\}=\left\{\begin{array}{l}
u_{0}(x, t)-z \frac{\partial w_{0}(x, t)}{\partial x} \\
w_{0}(x, t)+B \frac{\partial^{2} w_{0}(x, t)}{\partial x^{2}}
\end{array}\right\}
$$

In which $B$ becomes $B=\frac{D_{11}}{A_{44}}$.

The dynamic equilibrium of the model is derived with the calculus of variations on the basis of the Hamilton's principle leading to formulating governing equations [48] 


$$
\delta \Pi=\int_{0}^{t}(\delta \Omega-\delta S+\delta T) d t=0
$$

where $\Pi$ depicts total potential energy of the wire, $T$ and $\Omega$ denote the kinetic energy and the work done by outer loads. $S$ also symbolizes the strain energy of the wire.

\subsection{The strain potential energy}

The strain energy would be shown in a variational form as [48]

$$
\delta S=\iiint_{V}\left(\sigma_{i j} \delta \varepsilon_{i j}-D_{i} \delta E_{i}\right) d V=0
$$

In which the aforementioned parameters are respectively the electric field $\left(E_{i}\right)$, electric displacement $\left(D_{i}\right)$, strain tensor $\left(\varepsilon_{i j}\right)$, and stress tensor $\left(\sigma_{i j}\right)$, and can be summarized as follows [49]

$$
\begin{gathered}
\sigma_{i j}=C_{i j k l} \varepsilon_{k l}-e_{i j k} E_{k} \\
D_{i}=e_{i k l} \varepsilon_{k l}+\kappa_{i k} E_{k}
\end{gathered}
$$

where the displayed constants are piezoelectric $\left(e_{i j k}\right)$, dielectric $\left(\kappa_{i k}\right)$, and elasticity quantities $\left(C_{i j k l}\right)$. The tensors in Eqs. (4-5) lead to [49]:

$$
\begin{gathered}
e_{i j k}=\left\{\begin{array}{l}
\bar{e}_{31} \\
\bar{e}_{15}
\end{array}\right\} \\
\kappa_{i k}=\left\{\begin{array}{l}
\bar{\kappa}_{11} \\
\bar{\kappa}_{33}
\end{array}\right\} \\
\left\{\begin{array}{l}
\bar{e}_{31} \\
\bar{e}_{15} \\
\bar{\kappa}_{11} \\
\bar{\kappa}_{33}
\end{array}\right\}=\left\{\begin{array}{l}
e_{15}-\frac{C_{13} e_{33}}{C_{33}} \\
\kappa_{11} \\
\kappa_{33}+\frac{e_{33}^{2}}{C_{33}}
\end{array}\right\} \\
\varepsilon_{i j}=\frac{1}{2\left(\frac{\partial u_{i}}{\partial x_{j}}+\frac{\partial u_{j}}{\partial x_{i}}+\frac{\partial u_{k}}{\partial x_{i}} \frac{\partial u_{k}}{\partial x_{j}}\right) \Rightarrow} \\
\left\{\begin{array}{l}
\varepsilon_{x x} \\
\gamma_{x z}
\end{array}\right\}=\left\{\begin{array}{l}
\frac{\partial u_{0}}{\partial x}-z \frac{\partial^{2} w_{0}}{\partial x^{2}}+\frac{1}{2}\left(B \frac{\partial^{3} w_{0}}{\partial x^{3}}+\frac{\partial w_{0}}{\partial x}\right)^{2} \\
B \frac{\partial^{3} w_{0}}{\partial x^{3}}
\end{array}\right\}
\end{gathered}
$$

A potential function for the longitudinal electric field can be chosen as [49]. 


$$
\bar{\Phi}(x, z, t)=-\cos \left(\frac{\pi z}{d}\right) \Phi(x, t)+\frac{2 z V_{0}}{d} e^{i \omega t}
$$

In which $\Phi(x, t)$ denotes the electric field and the outer electric voltage is shown by $V_{0}$. Afterwards, the piezoelectric components take the form [49]:

$$
E_{i}=\left\{\begin{array}{l}
\bar{E}_{x} \\
\bar{E}_{z}
\end{array}\right\}=\left\{\begin{array}{l}
-\frac{\partial \bar{\Phi}(x, t)}{\partial x} \\
-\frac{\partial \bar{\Phi}(x, t)}{\partial z}
\end{array}\right\}=\left\{\begin{array}{l}
\cos \left(\frac{\pi z}{d}\right) \frac{\partial \Phi(x, t)}{\partial x} \\
-\frac{\pi}{d} \sin \left(\frac{\pi z}{d}\right) \Phi(x, t)-\frac{2 V_{0}}{d} e^{i \omega t}
\end{array}\right\}
$$

The electric displacements can be expressed as [49]:

$$
D_{i}=\left\{\begin{array}{l}
\bar{D}_{x} \\
\bar{D}_{z}
\end{array}\right\}=\left\{\begin{array}{l}
\int_{-d / 2}^{d / 2} D_{x} \cos \left(\frac{\pi z}{d}\right) d z \\
\int_{-d / 2}^{d / 2} \frac{\pi}{d} D_{z} \cos \left(\frac{\pi z}{d}\right) d z
\end{array}\right\}=\left\{\begin{array}{l}
E_{15} B \frac{\partial^{3} w_{0}}{\partial x^{3}}+X_{11} \frac{\partial \Phi}{\partial x} \\
-E_{31} \frac{\partial^{2} w_{0}}{\partial x^{2}}-X_{33} \Phi
\end{array}\right\}
$$

The coefficients in Eq. (12) are developed as

$$
\left\{\begin{array}{l}
E_{31} \\
E_{15} \\
X_{11} \\
X_{33}
\end{array}\right\}=\int_{-d / 2}^{d / 2}\left\{\begin{array}{c}
\bar{e}_{31} \frac{\pi}{d} z \sin \left(\frac{\pi}{d} z\right) \\
\bar{e}_{15} \cos \left(\frac{\pi}{d} z\right) \\
\bar{\kappa}_{11} \cos ^{2}\left(\frac{\pi}{d} z\right) \\
\bar{\kappa}_{33}\left(\frac{\pi}{d}\right)^{2} \sin ^{2}\left(\frac{\pi}{d} z\right)
\end{array}\right\} d z
$$

\subsection{The external force}

The Winkler model as an external force makes a thermodynamic work calculated as [48-49]

$$
\delta \Omega=-\iint_{A} k_{w} \frac{\partial^{2} w_{0}}{\partial x^{2}} \delta w_{0} d x=0
$$

where $k_{w}$ demonstrates the value of springiness in the foundation.

\subsection{The kinetic energy}

The kinetic energy is written as [48]

$$
T=\frac{1}{2} \rho \iint_{A}\left(\left(\frac{\partial U}{\partial t}\right)^{2}+\left(\frac{\partial W}{\partial t}\right)^{2}\right) d A d z=0
$$


Applying the variational form of the kinetic energy, one gets

$$
\begin{aligned}
& \delta T=\rho \iint_{A}\left(-\frac{\partial^{2} u_{0}}{\partial t^{2}}+z \frac{\partial^{3} w_{0}}{\partial x \partial t^{2}}\right) \delta u_{0}+\left(z \frac{\partial^{3} u_{0}}{\partial x \partial t^{2}}-z^{2}\left(\frac{\partial^{2} w_{0}}{\partial x \partial t}\right)^{2}\right. \\
& \left.-z^{2} \frac{\partial^{4} w_{0}}{\partial x^{2} \partial t^{2}}-\frac{\partial^{2} w_{0}}{\partial t^{2}}-B^{2} \frac{\partial^{6} w_{0}}{\partial x^{4} \partial t^{2}}-2 B \frac{\partial^{4} w_{0}}{\partial x^{2} \partial t^{2}}\right) \delta w_{0} d A d z=0
\end{aligned}
$$

where $I_{m}\left(I_{m}=\rho I_{c}\right)$ shows the mass moment of inertia, $m_{0}\left(m_{0}=\rho \int_{A} d A\right)$ is the volumetric mass density, and $\rho$ exhibits the sectional density.

Finally, doing $\delta \Pi=0$ gives problem equations as

$$
\begin{gathered}
\delta u_{0}: \frac{\partial N_{x}}{\partial x}=0 \\
\delta w_{0}:-\frac{\partial^{2} M_{x}}{\partial x^{2}}+B \frac{\partial^{3} Q_{x}}{\partial x^{3}}+N_{x}\left(B^{2} \frac{\partial^{6} w_{0}}{\partial x^{6}}+2 B \frac{\partial^{4} w_{0}}{\partial x^{4}}+\frac{\partial^{2} w_{0}}{\partial x^{2}}\right)-k_{w} \frac{\partial^{2} w_{0}}{\partial x^{2}} \\
-I_{m}\left(\frac{\partial^{4} w_{0}}{\partial x^{2} \partial t^{2}}\right)-m_{0} \times\left(\frac{\partial^{2} w_{0}}{\partial t^{2}}+B^{2} \frac{\partial^{6} w_{0}}{\partial x^{4} \partial t^{2}}+2 B \frac{\partial^{4} w_{0}}{\partial x^{2} \partial t^{2}}\right)=0 \\
\delta \Phi=0: \int_{-d / 2}^{d / 2}\left[\frac{\partial \bar{D}_{x}}{\partial x} \cos \left(\frac{\pi z}{d}\right)+\frac{\pi}{d} \bar{D}_{z} \sin \left(\frac{\pi z}{d}\right)\right] d z=0
\end{gathered}
$$

In which $N_{x}, M_{x}$ and $Q_{x}$ symbolize respectively the in-plane, moment and shear stress resultants.

The stress resultants are written as

$$
\left\{\begin{array}{c}
M_{x} \\
Q_{x}
\end{array}\right\}=\int_{A}\left\{\begin{array}{c}
\sigma_{x} z \\
\sigma_{x z}
\end{array}\right\} d A
$$

Therefore Eq. (18) is rewritten as

$$
\left\{\begin{array}{l}
M_{x} \\
Q_{x}
\end{array}\right\}=\left\{\begin{array}{c}
-D_{11} \frac{\partial^{2} w_{0}}{\partial x^{2}} \\
A_{44}\left(B \frac{\partial^{3} w_{0}}{\partial x^{3}}\right)
\end{array}\right\}+\left\{\begin{array}{c}
E_{31} \Phi \\
-E_{15} \frac{\partial \Phi}{\partial x}
\end{array}\right\}
$$

where the coefficients are

$$
\begin{gathered}
A_{44}=A \bar{C}_{44}, D_{11}=\bar{C}_{11} I_{c} \\
\left\{\begin{array}{l}
\bar{C}_{11} \\
\bar{C}_{44}
\end{array}\right\}=\left\{\begin{array}{l}
C_{11}-\frac{C_{13}^{2}}{C_{33}} \\
C_{44}
\end{array}\right\}
\end{gathered}
$$


$A$ reveals the nanowire' cross section. Furthermore, $I_{c}\left(I_{c}=\frac{\pi d^{4}}{64}\right)$ represents the area moment of the cross section.

In this research, axial stress resultant addresses the longitudinal electric load $\left(N_{i j}^{E}\right)$ created by the electric field as [49]:

$$
N_{x}^{E}=\int_{-d / 2}^{d / 2} e_{31} \frac{2 V_{0}}{d} d z
$$

The equation below is applied to address the nonlocal strain gradient theory (NSGT) [66]. Efficiency and accuracy of NSGT as a size-dependent model was approved in many papers and this nanoscale approach is now a well-known one. Therefore, in this research the NSGT is used corresponding to

$$
\begin{aligned}
& \left(1-\mu \nabla^{2}\right) \sigma_{i j}=C_{i j k l}\left(1-l^{2} \nabla^{2}\right) \varepsilon_{k l} ; \\
& \left\langle\mu\left(n m^{2}\right)=\left(e_{0} a\right)^{2}, \nabla^{2}=\frac{\partial^{2}}{\partial x^{2}}\right\rangle
\end{aligned}
$$

In which $\mu$ depicts nonlocality and also $l$ symbloizes a length scale factor for NSGT.

With regard to the Eq. (23) and applying it on the Eq. (19) the small scale stress resultants can be given by

$$
\begin{aligned}
& \left(1-\mu \nabla^{2}\right) M_{x}=-\left(1-l^{2} \nabla^{2}\right)\left(D_{11} \frac{\partial^{2} w_{0}}{\partial x^{2}}\right)+E_{31} \Phi \\
& \left(1-\mu \nabla^{2}\right) Q_{x}=\left(1-l^{2} \nabla^{2}\right) A_{44}\left(B \frac{\partial^{3} w_{0}}{\partial x^{3}}\right)-E_{15} \frac{\partial \Phi}{\partial x}
\end{aligned}
$$

The linear model of viscoelasticity, namely Kelvin-Voigt is here utilized to consider coupling of Viscous-Elasticity in the nanowire as [67]

$$
\sigma(t)=E(t) \varepsilon(t)=E\left(1+g \frac{\partial}{\partial t}\right) \varepsilon(t)
$$

In which the viscoelastic factor is represented by $g$. Now the equations below are achieved which include a combination of Eq. (17) with Eqs. (12), (22), (24) and (25). The obtained relations should be utilized in order to compute the vibration frequencies of the piezonanowire with viscoelasticity properties. 


$$
\begin{aligned}
& \left(1-l^{2} \nabla^{2}\right)\left[\left(1+g \frac{\partial}{\partial t}\right)\left(D_{11} \frac{\partial^{4} w_{0}}{\partial x^{4}}+A_{44} B^{2} \frac{\partial^{6} w_{0}}{\partial x^{6}}\right)-\left(B E_{15}+E_{31}\right) \frac{\partial^{2} \Phi}{\partial x^{2}}-\right. \\
& \left(1-\mu \nabla^{2}\right) N_{x}^{E}\left(B^{2} \frac{\partial^{6} w_{0}}{\partial x^{6}}+2 B \frac{\partial^{4} w_{0}}{\partial x^{4}}+\frac{\partial^{2} w_{0}}{\partial x^{2}}\right)+\left(1-\mu \nabla^{2}\right) \times \\
& {\left[-I_{m}\left(\frac{\partial^{4} w_{0}}{\partial x^{2} \partial t^{2}}\right)-m_{0}\left(\frac{\partial^{2} w_{0}}{\partial t^{2}}+B^{2} \frac{\partial^{6} w_{0}}{\partial x^{4} \partial t^{2}}+2 B \frac{\partial^{4} w_{0}}{\partial x^{2} \partial t^{2}}\right)-k_{w} \frac{\partial^{2} w_{0}}{\partial x^{2}}\right]=0} \\
& A_{44} B E_{15} \frac{\partial^{4} w_{0}}{\partial x^{4}}-D_{11} E_{31} \frac{\partial^{2} w_{0}}{\partial x^{2}}-X_{11} \frac{\partial^{2} \Phi}{\partial x^{2}}+X_{33} \Phi=0
\end{aligned}
$$

\section{Solution Technique}

In this section Navier solution method is used which reduces the partial differential equation (PDE) to an algebraic one as [48]

$$
\begin{gathered}
w_{0}(x, t)=y(x) W(t) \\
\Phi(x, t)=y(x) \Omega(t)
\end{gathered}
$$

In which $y(x)$ is a fundamental mode shape, $W(t)$ and $\Omega(t)$ are temporary functions based on time. The mode shape which determines pivot boundary conditions is as

$$
y(x)=\sin \left(\pi \frac{x}{L}\right)
$$

Substituting Eq. (27) into Eq. (26), Eq. (26) reduces to an algebraic equation where to compute pivot boundary supports, Eq. (28) is employed. Thereafter, in order to present vibration frequencies a harmonic function is assumed as below

$$
\begin{aligned}
\Omega(t) & =\exp \left(\omega_{n} t\right) \\
W(t) & =\exp \left(\omega_{n} t\right)
\end{aligned}
$$

In which $\omega_{n}$ corresponds to the complex frequency of the nanowire. The natural frequency is divided into two parts, real and imaginary as

$$
\omega_{n}=\lambda+i \chi, i=\sqrt{-1}
$$

In which $\lambda$ is the real part and $\chi$ is the imaginary part of the complex frequency, respectively. The real part shows damping ratio for the model and the imaginary part represents natural frequency. Consequently, based on the given algorithm and some mathematical simplifying, the equation below can be obtained.

$$
[K] \cdot\left\{\begin{array}{l}
W(t) \\
\Omega(t)
\end{array}\right\} \cdot \sin \left(\pi \frac{x}{L}\right) \cdot \exp \left(\omega_{n} t\right)=0
$$


To compute the natural frequency of the piezo-visco-nanowire, a nontrivial solution can be done by vanishing determinant of the coefficients matrix $(\operatorname{det}[K]=0)$. After that, by calculating the obtained equation based on $\omega_{n}$ the numeric outcomes for the natural frequency can be shown (Appendix A).

\section{Examples and Discussions}

At this point, several samples are considered with which a crystal comparison between the current formulation and others is presented. Table 1 considers several references with various beam approaches. As it is found, the numerical outcomes of the present work are matched with those obtained by references. In addition, very good agreements are observed whenever the beam tends to be thinner with increasing its ratio of length to thickness. The reason is because at this condition the influence of shear deformations cannot be important and the results of the mentioned beam theories are close to one another. By this Table, the current formulation can be approved and so, the numerical outcomes can be further developed by changes in the essential variables. On the other hand, the mechanical and electrical quantities and properties of the employed nanowire can be seen at Table 2 which are found by the wellknown references.

Table 1. Validations for nondimensional vibration frequencies.

\begin{tabular}{|c|c|c|c|c|c|c|}
\hline \multirow[t]{2}{*}{$L / h$} & \multirow[t]{2}{*}{$\left(e_{0} a\right)^{2}$} & \multirow[t]{2}{*}{ Present } & \multicolumn{2}{|c|}{$\begin{array}{l}\text { Timoshenko beam } \\
\text { theory (TBT) }\end{array}$} & \multicolumn{2}{|c|}{$\begin{array}{l}\text { Sinusoidal beam } \\
\text { theory (SBT) }\end{array}$} \\
\hline & & & [68] & [69] & [68] & [69] \\
\hline \multirow{5}{*}{5} & 0 & 9.2943 & 9.2740 & 9.2740 & 9.2752 & 9.2752 \\
\hline & 1 & 8.8587 & 8.8477 & 8.8477 & 8.8488 & 8.8488 \\
\hline & 2 & 8.4788 & 8.4752 & 8.4752 & 8.4763 & 8.4763 \\
\hline & 3 & 8.1495 & 8.1461 & 8.1461 & 8.1472 & 8.1472 \\
\hline & 4 & 7.8693 & 7.8526 & 7.8526 & 7.8536 & 7.8536 \\
\hline \multirow{5}{*}{10} & 0 & 9.7209 & 9.7075 & 9.7075 & 9.7077 & 9.7077 \\
\hline & 1 & 9.2666 & 9.2612 & 9.2612 & 9.2614 & 9.2614 \\
\hline & 2 & 8.8857 & 8.8713 & 8.8713 & 8.8715 & 8.8715 \\
\hline & 3 & 8.5483 & 8.5269 & 8.5269 & 8.5271 & 8.5271 \\
\hline & 4 & 8.2320 & 8.2196 & 8.2196 & 8.2198 & 8.2198 \\
\hline \multirow{5}{*}{20} & 0 & 9.8377 & 9.8281 & 9.8281 & 9.8282 & 9.8282 \\
\hline & 1 & 9.3840 & 9.3763 & 9.3763 & 9.3764 & 9.3764 \\
\hline & 2 & 8.9917 & 8.9816 & 8.9816 & 8.9816 & 8.9816 \\
\hline & 3 & 8.6456 & 8.6328 & 8.6328 & 8.6329 & 8.6329 \\
\hline & 4 & 8.3329 & 8.3218 & 8.3218 & 8.3218 & 8.3218 \\
\hline
\end{tabular}


Table 2. The mechanical, electrical and geometrical characteristics of the piezo-nanowire [49]

\begin{tabular}{cc}
\hline Material & Mechanical and electrical Properties \\
\hline & Dielectric $(C / V . m)$ \\
\cline { 2 - 2 } BaTiO $_{3}$ & $\kappa_{11}=5.64 e-9, \kappa_{33}=6.35 e-9$ \\
\cline { 2 - 2 } & Piezoelectric $\left(\mathrm{C} / \mathrm{m}^{2}\right)$ \\
\cline { 2 - 2 } & $e_{31}=-2.2, e_{15}=5.8, e_{33}=9.3$ \\
\cline { 2 - 2 } & Elastic $(\mathrm{GPa})$ \\
\hline$C_{11}=226, C_{13}=124$, \\
$C_{33}=216, C_{44}=44.2$ \\
\hline
\end{tabular}

According to Figs. 2, the changes in the parameter of the electric voltage can be seen versus small scale parameters' changes. It is evident from both figures the influences of changes in the outer voltage on frequency results of the system is insignificant. As a matter of fact, the nanowire used in the present study does not have a significant reaction to external electricity, although the influence is sufficiently large at such nanoscale. Perhaps it's because of its very low nanoscale thickness or its one dimensional manner. Furthermore, it is observed that increasing the nonlocal parameter in NSGT relation results in reduction of the natural frequency and regarding the second figure, the length scale parameter of the relation leads to increasing of the natural frequency. It can also be worth noting that in the case of both parameters which have the same values (e.g. value 1), the natural frequency of the nanowire in both figures will be in same values that are quite logical. In fact, this mode represents a local analysis, not taking the influences of small scale into account.

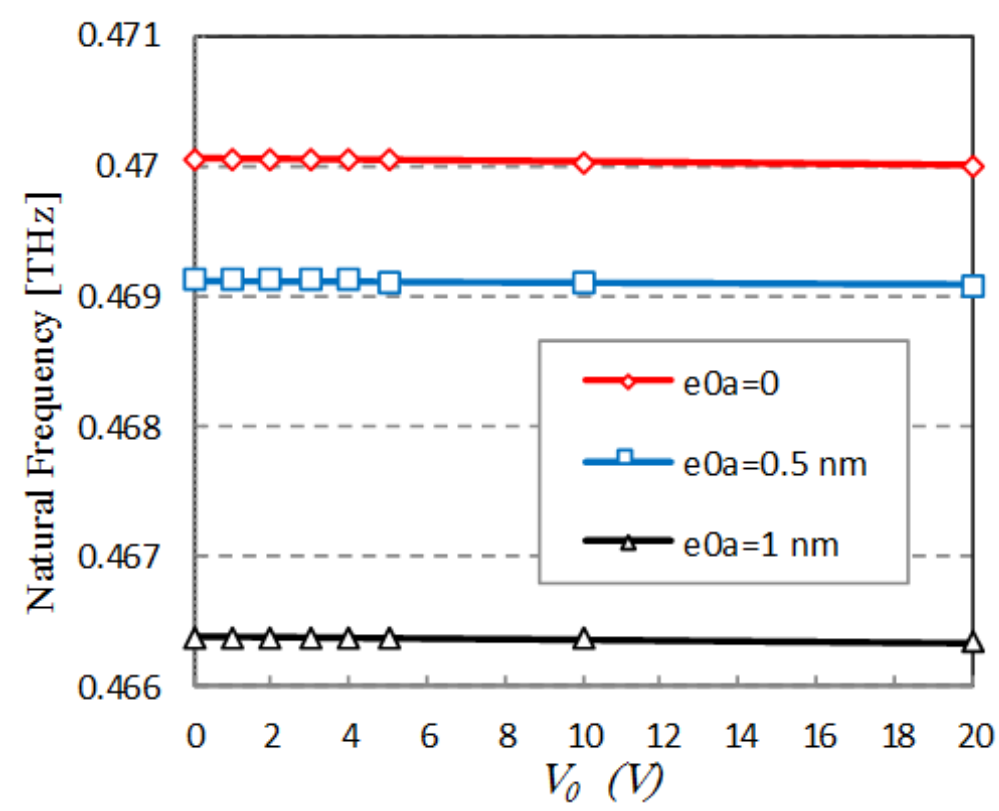

Fig. 2a. Effects of variations of the electric voltage versus nonlocal parameter on the natural frequencies $(\beta=5, l=1 \mathrm{~nm}, g=5 \mathrm{~N} . \mathrm{s} / \mathrm{m})$ 


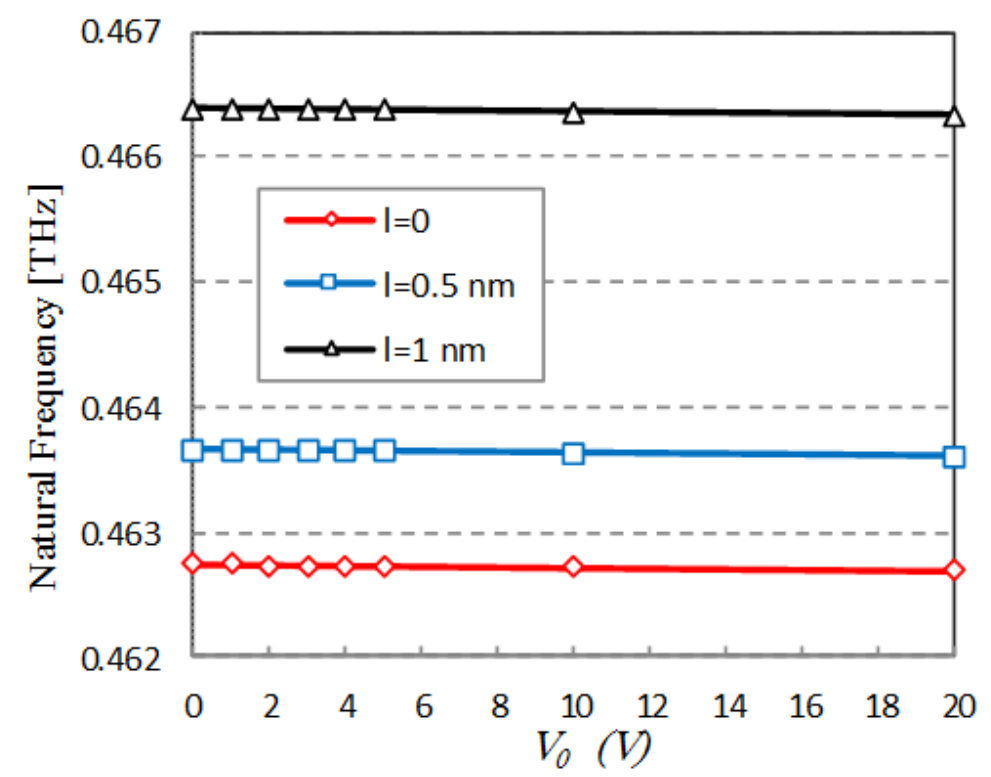

Fig. 2b. Effects of variations of the electric voltage versus length scale parameter on the natural frequencies $\left(\beta=5, e_{0} a=1 \mathrm{~nm}, g=5 \mathrm{~N} . \mathrm{s} / \mathrm{m}\right)$

Figure 3 exhibits the variation of the coefficient of internal viscosity of the nanowire against the nonlocal coefficient's variation. It is quite clear from the figure that the natural frequency is increased with increasing the viscoelastic coefficient. In fact, from a physical point of view, by increasing the coefficient, the nanowire's energy absorption is higher, and so the nanowire will have a larger frequency. Moreover, the frequency variations are linear and with a slight gradient. It can be concluded that for nanowires with very large lengths, the effect of viscosity is not remarkable. In Fig. 4, the effect of changes in the Winkler elastic foundation on the frequency results of the nanowire can be observed. In fact, after embedding the elastic base, the system provides greater frequencies. Such an increase is shown with a fairly significant gradient in the aforementioned figure. It can also be seen that changes in the viscosity of the nanowire do not affect the variation of the elastic base. The reason is as a result of the parallelism of results of the three viscosity coefficients in Fig. 4.

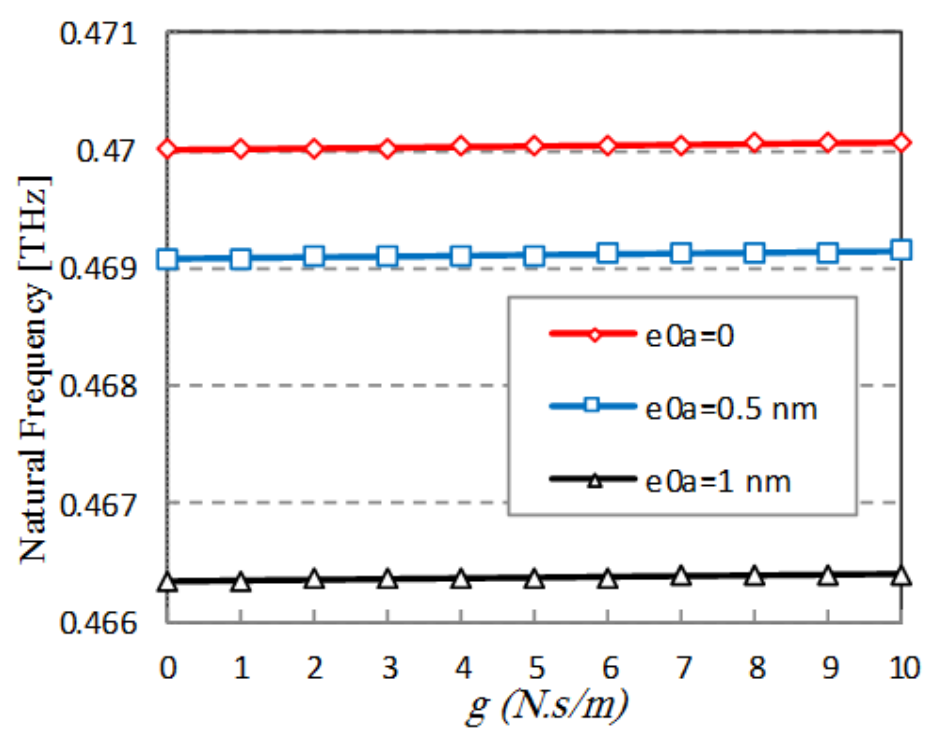

Fig. 3. Effects of variations of the viscoelastic coefficient versus nonlocal parameter on the natural frequencies $\left(V_{0}=5 \mathrm{~V}, \beta=5, l=1 \mathrm{~nm}\right)$ 


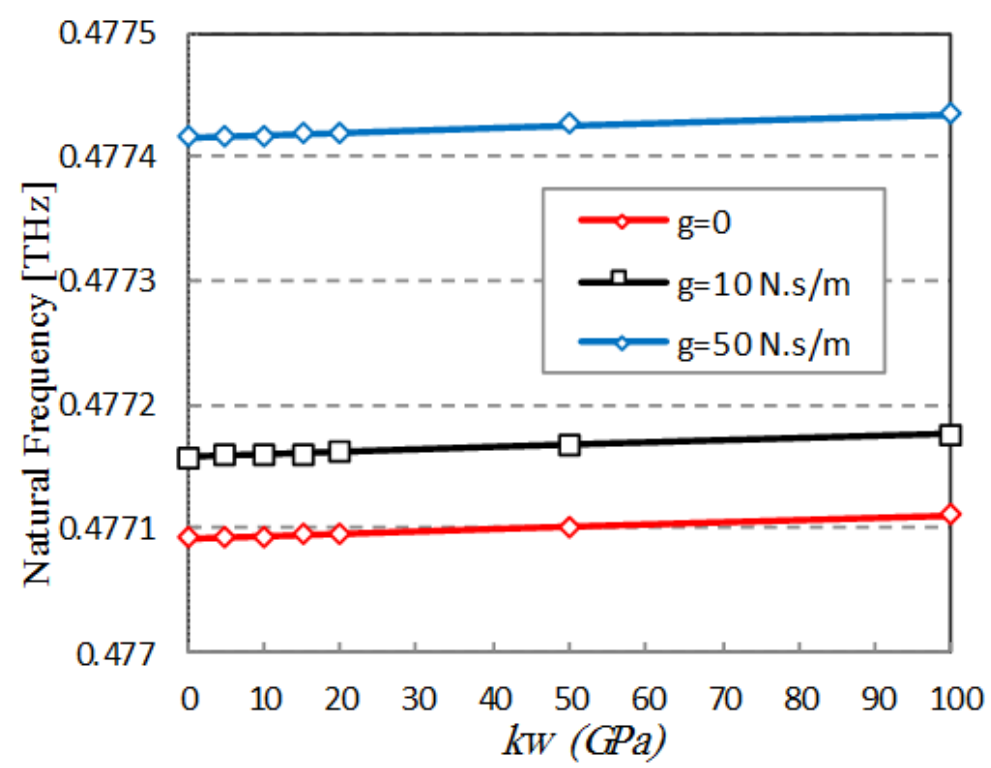

Fig. 4. Effects of variations of the Winkler parameter versus viscoelastic coefficient on the natural frequencies $\left(V_{0}=5 \mathrm{~V}, \beta=5, e_{0} a=1 \mathrm{~nm}, l=2 \mathrm{~nm}\right)$

Figure 5 shows an important effect on nanowires. In fact, the physical nature of the nanowires is wires of very long lengths. For example, nanowires with a length to thickness ratio of 1,000 are also available. According to this figure, it can be seen that in large proportions of this coefficient, the small scale effect is completely unimportant. However, in small ratios of this factor, the effect of small scale will be larger. Additionally, increasing the aspect ratio to 7 will lead to a sharp decrease of vibration results for the nanostructure, and then the slope of the results will be mitigated. To the extent that it can be said, in very large quantities, the effect of the coefficient's changes does not affect the frequency results of the modeled system in the present study.

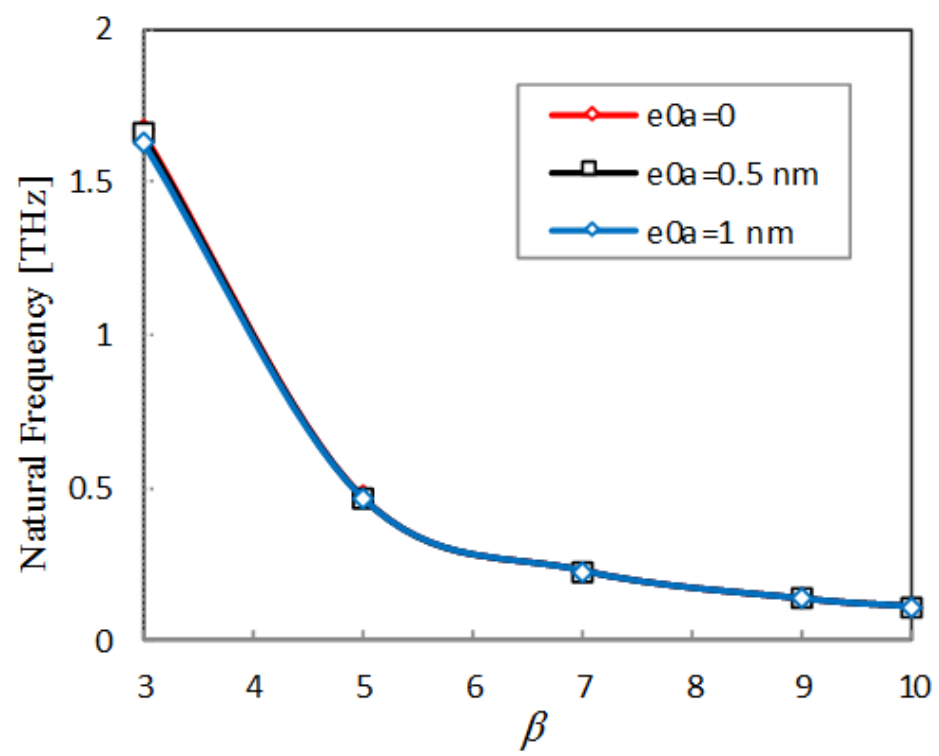

Fig. 5. Effects of variations of the aspect ratio versus nonlocal parameter on the natural frequencies $\left(V_{0}=10 \mathrm{~V}, g=5 \mathrm{~N} . \mathrm{s} / \mathrm{m}, \mathrm{l}=1 \mathrm{~nm}\right)$ 


\section{Conclusions}

In the study, a nanowire was embedded in an elastic substrate, and the electric field was assumed to be longitudinal. To analyze the effects of size-dependent, the nonlocal strain gradient theory was employed which has two variables. One parameter, known as nonlocal parameter, measures effects of quantum mechanics on the surface, and the second factor, known as the length scale one, measures the stiffness effects of the material by decreasing its size. The marked outcomes are listed below:

- Although the $\mathrm{BaTiO}_{3}$ nanowire does not react remarkably to the external voltage, the influence is sufficiently large and cannot be neglected at nanoscale.

- Whilst the nanowire is very large, the effects of small scale and viscoelasticity cannot be considerable.

- The effect of increase of the external voltage on the nonlocal parameter is more than the length scale one.

- Increase of the viscoelastic parameter is further remarkable for lower values of the nonlocal parameter.

\section{Appendix A:}

$$
\begin{aligned}
& K_{11}=\left\{\left[\left(1+g \omega_{n}\right)\left(D_{11}\left(\frac{\pi}{L}\right)^{4}+A_{44} B^{2}\left(-\left(\frac{\pi}{L}\right)^{6}\right)\right)\right]+N_{x}^{E}\left(B^{2}\left(-\left(\frac{\pi}{L}\right)^{6}\right)+2 B\left(\frac{\pi}{L}\right)^{4}+\left(-\left(\frac{\pi}{L}\right)^{2}\right)\right)+\left(\omega_{n}\right)^{2} \times\right. \\
& \left.\left[-I_{m}\left(-\left(\frac{\pi}{L}\right)^{2}\right)-m_{0}\left(1+B^{2}\left(\frac{\pi}{L}\right)^{4}+2 B\left(-\left(\frac{\pi}{L}\right)^{2}\right)\right)\right]\right\}+\left\{-l^{2}\left[\left(1+g \omega_{n}\right)\left(D_{11}\left(-\left(\frac{\pi}{L}\right)^{6}\right)+A_{44} B^{2}\left(\frac{\pi}{L}\right)^{8}\right)\right]\right. \\
& -k_{w}\left(-\left(\frac{\pi}{L}\right)^{2}\right)-\mu N_{x}^{E}\left(B^{2}\left(\frac{\pi}{L}\right)^{8}+2 B\left(-\left(\frac{\pi}{L}\right)^{6}\right)+\left(\frac{\pi}{L}\right)^{4}\right)+\mu k_{w}\left(\frac{\pi}{L}\right)^{4}-\mu\left(\omega_{n}\right)^{2} \times \\
& \left.\left[-I_{m}\left(\frac{\pi}{L}\right)^{4}-m_{0}\left(\left(-\left(\frac{\pi}{L}\right)^{2}\right)+B^{2}\left(-\left(\frac{\pi}{L}\right)^{6}\right)+2 B\left(\frac{\pi}{L}\right)^{4}\right)\right]\right\} \\
& K_{12}=\left(B E_{15}+E_{31}\right)\left(\frac{\pi}{L}\right)^{2}+l^{2}\left(B E_{15}+E_{31}\right)\left(\frac{\pi}{L}\right)^{4} \\
& K_{21}=A_{44} E_{15}\left(\frac{\pi}{L}\right)^{4}-D_{11} E_{31}\left(-\left(\frac{\pi}{L}\right)^{2}\right) \\
& K_{22}=-X_{11}\left(-\left(\frac{\pi}{L}\right)^{2}\right)+X_{33}
\end{aligned}
$$




\section{Acknowledgments}

The authors thank to the reviewers for their valuable comments which lead to improving the manuscript.

\section{References}

[1] Rao, C. N. R., Govindaraj, A., Nanotubes and Nanowires. First Publishing, UK, R. S. C. Publication, 2005.

[2] Hu, J., Odom, T. W., Lieber, C. M., Chemistry and Physics in One Dimension: Synthesis and Properties of Nanowires and Nanotubes. Accounts of Chemical Research, 32, 435445, 1999.

[3] Lupu, N., Nanowires Science and Technology. First Publishing, India, Intech, 2010.

[4] Wang, Z. L., Nanowires and Nanobelts: Materials Properties and Devices, Nanowires and Nanobelts of Functional Materials. First printing, USA, Springer, 2006.

[5] Kiani, K., Magneto-elasto-dynamic analysis of an elastically confined conducting nanowire due to an axial magnetic shock. Physics Letters A, 376, 1679-1685, 2012.

[6] Kiani, K., Dynamic interactions between double current-carrying nanowires immersed in a longitudinal magnetic field: Novel integro-surface energy-based models. International Journal of Engineering Science, 107, 98-133, 2016.

[7] Pishkenari, H. N., Afsharmanesh, B., Tajaddodianfar, F., Continuum models calibrated with atomistic simulations for the transverse vibrations of silicon nanowires. International Journal of Engineering Science, 100, 8-24, 2016.

[8] Fu, Y., Zhang, P., Buckling and vibration of core-shell nanowires with weak interfaces. Mechanics Research Communications, 37, 622-626, 2010.

[9] Kiani, K., Surface effect on free transverse vibrations and dynamic instability of currentcarrying nanowires in the presence of a longitudinal magnetic field. Physics Letters A, 378, 1834-1840, 2014.

[10] Gongbai, C., Yunfei, Ch., Jiwei, J., Yuelin, W., Harmonic behavior of silicon nanowire by molecular dynamics. Mechanics Research Communications, 34, 503-507, 2007.

[11] Zhoua, J., Wanga, Zh., Grotsb, A., Heb, X., Electric field drives the nonlinear resonance of a piezoelectric nanowire. Solid State Communications, 144, 118-123, 2007.

[12] Zhang, Y. Q., Pang, M., Chen, W. Q., Transverse vibrations of embedded nanowires under axial compression with high-order surface stress effects. Physica E, 66, 238-244, 2015

[13] Li, X.-F., Wang, B.-L., Tang, G.-J., Lee, K.-Y., Size effect in transverse mechanical behavior of one-dimensional nanostructures. Physica E, 44, 207-214, 2011.

[14] Su, G.-Y., Li, Y.-X., Li, X.-Y., Muller, R., Free and forced vibrations of nanowires on elastic substrates. International Journal of Mechanical Sciences, 138-139, 62-73, 2018. 
[15] Tourki Samaei, A., Gheshlaghi, B., Wang, G.-F., Frequency analysis of piezoelectric nanowires with surface effects. Current Applied Physics, 13, 2098-2102, 2013.

[16] Gheshlaghi, B., Hasheminejad, S. M., Vibration analysis of piezoelectric nanowires with surface and small scale effects. Current Applied Physics, 12, 1096-1099, 2012.

[17] Kiani, K., Forced vibrations of a current-carrying nanowire in a longitudinal magnetic field accounting for both surface energy and size effects. Physica E: Low-Dimensional Systems and Nanostructures, 63, 27-35, 2014.

[18] Kiani, K., Stability and vibrations of doubly parallel current-carrying nanowires immersed in a longitudinal magnetic field. Physics Letters A, 379, 348-360, 2015.

[19] Kiani, K., A refined integro-surface energy-based model for vibration of magnetically actuated doublenanowire- systems carrying electric current. Physica E: Low-Dimensional Systems and Nanostructures, 86, 225-236, 2017.

[20] Mercan, K., Numanoglu, H. M., Akgöz, B., Demir, C., \& Civalek, Ö., Higher order continuum theories for buckling response of silicon carbide nanowires (SiCNWs) on elastic matrix. Archive of Applied Mechanics, 87, 1797-1814, 2017.

[21] Mercan, K., \& Civalek, Ö., Modal Analysis of Micro and Nanowires Using Finite Element Softwares. International Journal of Engineering \& Applied Sciences, 10, 291304, 2018.

[22] Numanoglu, H. M., Mercan, K., \& Civalek, Ö., Frequency and mode shapes of Au nanowires using the continuous beam models. International Journal of Engineering and Applied Sciences, 4, 55-61, 2017.

[23] Akgöz, B., and Civalek, Ö., A size-dependent shear deformation beam model based on the strain gradient elasticity theory. International Journal of Engineering Science, 70, 114, 2013.

[24] Avcar, M., Free vibration of imperfect sigmoid and power law functionally graded beams. Steel and Composite Structures, 30, 603-615, 2019.

[25] Avcar, M., Effects of rotary inertia shear deformation and non-homogeneity on frequencies of beam. Structural Engineering and Mechanics, 55, 871-884, 2015.

[26] Avcar, M., Free vibration analysis of beams considering different geometric characteristics and boundary condition. International Applied Mechanics, 4, 94-100, 2014.

[27] Numanoglu, H. M., Akgöz, B., Civalek, Ö., On dynamic analysis of nanorods. International Journal of Engineering Science, 130, 33-50, 2018.

[28] Ufuk, G., Aydogdu, M., Noncoaxial vibration and buckling analysis of embedded double-walled carbon nanotubes by using doublet mechanics. Composites Part B: Engineering, 137, 60-73, 2018.

[29] Demir, C., Civalek, Ö., On the analysis of microbeams. International Journal of Engineering Science, 121, 14-33, 2017. 
[30] Zhang, B., He, Y., Liu, D., Gan, Z., Shen, L., Non-classical Timoshenko beam element based on the strain gradient elasticity theory. Finite Elements in Analysis and Design, 79, 22-39, 2014.

[31] Mercan, K., Civalek, Ö., DSC method for buckling analysis of boron nitride nanotube (BNNT) surrounded by an elastic matrix. Composite Structures, 143, 300-309, 2016.

[32] Barretta, R. et al., A higher-order Eringen model for Bernoulli-Euler nanobeams. Archive of Applied Mechanics, 86, 483-495, 2016.

[33] Akgöz, B, Civalek Ö., Effects of thermal and shear deformation on vibration response of functionally graded thick composite microbeams. Composites Part B: Engineering, 129, 77-87, 2017.

[34] Mercan, K., Civalek, Ö., Buckling analysis of Silicon carbide nanotubes (SiCNTs) with surface effect and nonlocal elasticity using the method of HDQ. Composites Part B, 114, 34-45, 2017.

[35] Rahmani, O., Refaeinejad, V., Hosseini, S. A. H., Assessment of various nonlocal higher order theories for the bending and buckling behavior of functionally graded nanobeams. Steel \& Composite Structures, An International Journal, 23, 339-350, 2014.

[36] Akgöz, B., Civalek, Ö., Buckling analysis of cantilever carbon nanotubes using the strain gradient elasticity and modified couple stress theories. Journal of Computational and Theoretical Nanoscience, 8, 1821-1827, 2011.

[37] Demir, C., Mercan, K., Civalek, Ö., Determination of critical buckling loads of isotropic, FGM and laminated truncated conical panel. Composites Part B, 94, 1-10, 2016.

[38] Civalek, Ö., Free vibration of carbon nanotubes reinforced (CNTR) and functionally graded shells and plates based on FSDT via discrete singular convolution method. Composites Part B: Engineering, 111, 45-59, 2017.

[39] Akgöz, B., Civalek, Ö., Nonlinear vibration analysis of laminated plates resting on nonlinear two parameters elastic foundations. Steel and Composite Structures, 11, 403 421, 2011.

[40] Thai, H. T., Kim, S. E., A review of theories for the modeling and analysis of functionally graded plates and shells. Composite Structures, 128, 70-86, 2015.

[41] Civalek, Ö., Acar M. H., Discrete singular convolution method for the analysis of Mindlin plates on elastic foundations. International Journal of Pressure Vessels and Piping, 84, 527-535, 2007.

[42] Shirmohammadi, F., Bahrami, S., Dynamic response of circular and annular circular plates using spectral element method. Applied Mathematical Modelling, 53, 156-166, 2018.

[43] Mercan, K., Civalek, Ö., DSC method for buckling analysis of boron nitride nanotube (BNNT) surrounded by an elastic matrix. Composite Structures, 143, 300-309, 2016. 
[44] Civalek, Ö., Demir, Ç., Bending analysis of microtubules using nonlocal Euler-Bernoulli beam theory. Applied Mathematical Modelling, 35, 2053-2067, 2011.

[45] Demir, Ç., Civalek, Ö., Akgöz, B., Free vibration analysis of carbon nanotubes based on shear deformable beam theory by discrete singular convolution technique. Mathematical and Computational applications, 15, 57-65, 2010.

[46] Demir, Ç., Civalek, Ö., Akgöz, B., Free vibration analysis of carbon nanotubes based on shear deformable beam theory by discrete singular convolution technique. Mathematical and Computational applications, 15, 57-65, 2010.

[47] Malikan, M., Electro-mechanical shear buckling of piezoelectric nanoplate using modified couple stress theory based on simplified first order shear deformation theory. Applied Mathematical Modelling, 48, 196-207, 2017.

[48] Malikan, M., Nguyen, V. B., Tornabene, F., Damped forced vibration analysis of singlewalled carbon nanotubes resting on viscoelastic foundation in thermal environment using nonlocal strain gradient theory, Engineering Science and Technology, an International Journal, 21, 778-786, 2018.

[49] Malikan, M., Nguyen, V. B., Buckling analysis of piezo-magnetoelectric nanoplates in hygrothermal environment based on a novel one variable plate theory combining with higher-order nonlocal strain gradient theory. Physica E: Low-Dimensional Systems and Nanostructures, 102, 8-28, 2018.

[50] Malikan, M., Temperature influences on shear stability of a nanosize plate with piezoelectricity effect. Multidiscipline Modeling in Materials and Structures, 14, 125$142,2018$.

[51] Malikan, M., Electro-thermal buckling of elastically supported double-layered piezoelectric nanoplates affected by an external electric voltage. Multidiscipline Modeling in Materials and Structures, 15, 50-78, 2019.

[52] Malikan, M., Buckling analysis of a micro composite plate with nano coating based on the modified couple stress theory. Journal of Applied and Computational Mechanics, 4, $1-15,2018$.

[53] Malikan, M., On the buckling response of axially pressurized nanotubes based on a novel nonlocal beam theory. Journal of Applied and Computational Mechanics, 5, 103-112, 2019.

[54] Malikan, M., Jabbarzadeh, M., Dastjerdi, Sh., Non-linear Static stability of bi-layer carbon nanosheets resting on an elastic matrix under various types of in-plane shearing loads in thermo-elasticity using nonlocal continuum. Microsystem Technologies, 23, 2973-2991, 2017.

[55] Malikan, M., Nguyen, V.B., A novel one-variable first-order shear deformation theory for biaxial buckling of a size-dependent plate based on the Eringen's nonlocal differential law. World Journal of Engineering, 15, 633-645, 2018.

[56] Malikan, M., Sadraee Far, M. N., Differential quadrature method for dynamic buckling of graphene sheet coupled by a viscoelastic medium using neperian frequency based on 
nonlocal elasticity theory. Journal of Applied and Computational Mechanics, 4, 147-160, 2018.

[57] Malikan, M., Tornabene, F., Dimitri, R., Nonlocal three-dimensional theory of elasticity for buckling behavior of functionally graded porous nanoplates using volume integrals. Materials Research Express, 5, 095006, 2018.

[58] Malikan, M., Nguyen, V.B., Tornabene, F., Electromagnetic forced vibrations of composite nanoplates using nonlocal strain gradient theory. Materials Research Express, 5, 075031, 2018.

[59] Malikan, M., Dimitri, R., Tornabene, F., Effect of Sinusoidal Corrugated Geometries on the Vibrational response of Viscoelastic Nanoplates. Applied Sciences, 8, 1432, 2018.

[60] Malikan, M., Nguyen, V.B., Dimitri, R., Tornabene, F., Dynamic modeling of noncylindrical curved viscoelastic single-walled carbon nanotubes based on the second gradient theory. Materials Research Express, 6, 075041, 2019.

[61] Malikan, M., Dimitri, R., Tornabene, F., Transient response of oscillated carbon nanotubes with an internal and external damping. Composites Part B: Engineering, 158, 198-205, 2019.

[62] Malikan, M., Analytical predictions for the buckling of a nanoplate subjected to nonuniform compression based on the four-variable plate theory. Journal of Applied and Computational Mechanics, 3, 217-228, 2017.

[63] Malikan, M., Dastjerdi, Sh., Analytical buckling of FG nanobeams on the basis of a new one variable first-order shear deformation beam theory. International Journal of Engineering \& Applied Sciences, 10, 21-34, 2018.

[64] Golmakani, M. E., Malikan, M., Sadraee Far, M. N., Majidi, H. R., Bending and buckling formulation of graphene sheets based on nonlocal simple first order shear deformation theory, Materials Research Express, 5, 065010, 2018.

[65] Malikan, M., Dimitri, R., Tornabene, F., Thermo-resonance analysis of an excited graphene sheet using a new approach, International Journal of Engineering \& Applied Sciences, 10, 190-206, 2018.

[66] Lim, C. W., Zhang, G., Reddy, J. N., A Higher-order nonlocal elasticity and strain gradient theory and Its Applications in wave propagation. Journal of the Mechanics and Physics of Solids, 78, 298-313, 2015.

[67] Lei, Y., Adhikari, S., Friswell, M. I., Vibration of nonlocal Kelvin-Voigt viscoelastic damped Timoshenko beams. International Journal of Engineering Science, 66-67, 1-13, 2013.

[68] Thai, H. T., A nonlocal beam theory for bending, buckling, and vibration of nanobeams, International Journal of Engineering Science, 52, 56-64, 2012.

[69] Lu, L., Guo, X., Zhao, J., Size-dependent vibration analysis of nanobeams based on the nonlocal strain gradient theory. International Journal of Engineering Science, 116, 12 24, 2017. 